\title{
Improvements in Bone Pain and Patient Quality of Life in Adults with Gaucher Disease During Substrate Reduction Therapy: A Case Series
}

\section{Amato $D^{*}$, Patterson MA}

Mark Freedman and Judy Jacobs Program for Gaucher Disease, Mount Sinai Hospital and University of Toronto, Toronto, Canada

\begin{abstract}
Background: Gaucher disease type 1 (GD1) is an inherited lysosomal storage disorder caused by a deficiency of glucocerebrosidase, and is characterised by haematological, visceral and bone manifestations that vary widely in terms of severity and age at onset. In particular, the bone manifestations of GD1 can restrict physical activity and impact heavily on patient quality of life (QoL). Here, we describe three GD1 patients who showed remarkable improvements in bone-related outcomes during treatment with oral miglustat, two of whom had previously failed to respond to relatively high doses of enzyme replacement therapy (ERT). In the third, needle phobia led to oral miglustat being used as the initial treatment.
\end{abstract}

Case presentation: Case 1 is a 39 -year-old female diagnosed aged 4 years (genotype N370S/L444P) and underwent ERT (alglucerase then imiglucerase) since 1994. In 2013 she discontinued ERT (treatment duration 19 years) due to ongoing and debilitating bone pain and fatigue and switched to miglustat therapy. She has since experienced substantial reductions in bone pain and improved physical function. Case 2 is a 59-year-old female diagnosed aged 17 years (genotype N370S/H162P). She commenced ERT treatment in 2002, with a brief interruption in 2007, and continued on ERT (imiglucerase followed by velaglucerase) up to 2014: total ERT monotherapy duration 12 years. She subsequently received combination therapy (velaglucerase plus miglustat) for 2 years but finally switched to miglustat monotherapy in 2016 due to ongoing bone manifestations. Her bone pain has since improved a great deal, alongside improvements in QoL parameters. Case 3 is a 48-year-old male diagnosed aged 11 years (genotype R463C/L105R). Having not received any previous treatment he avoided ERT due to life-long needle phobia. He therefore started miglustat therapy in late-2017 and has received it for approximately 1 year. During this time, he experienced vast improvements in QoL due to decreased bone pain. Interestingly in all three cases, there were little or no observed changes in objective bone parameters (bone marrow burden, bone mineral density).

Conclusions: These three GD1 cases illustrate the potential to achieve substantial reductions in bone pain and improvements in
QoL, even in patients who have failed to respond to long-term ERT regarding bone status.

\section{Background}

Gaucher disease (GD) is an inherited lysosomal storage disorder caused by a deficiency of the enzyme glucocerebrosidase. The commonest clinical manifestations of GD type 1 (GD1) are hepatosplenomegaly, thrombocytopenia, anaemia, and bone pain. In particular, the bone manifestations of GD can impact heavily on patient quality of life and can restrict physical activities [1,2]. The most common bone manifestations include bone pain, osteopenia, Erlenmeyer-flask deformity, increased risk of fractures, bone crises, and avascular necrosis $[2,3]$.

Intravenous enzyme replacement therapies (ERT) and oral substrate reduction therapies (SRT) are available to treat GD1. ERTs include imiglucerase (Cerezyme ${ }^{\circledast}$; Sanofi-Genzyme), velaglucerase (Vpriv ${ }^{\circledR}$; Shire Human Genetics) and taliglucerase (Elelyso ${ }^{\circledR}$; Pfizer/Protalix) and are usually administered every two weeks [4]. Available SRTs comprise miglustat (Zavesca ${ }^{\oplus}$; Actelion Pharmaceuticals) and eliglustat (Cerdelga ; Sanofi-Genzyme), and are taken as oral capsules each day. All of these treatments have been shown to treat core visceral and haematological symptoms of GD1 [4-6], but their effects on bone manifestations remain less well defined as they often require long periods of treatment and, in the case of ERT, higher dos-

Correspondence: Dominick Amato, Department of Medicine, Mount Sinai Hospital, 60 Murray Street, Room L-315, Box 34, Toronto, ON, M5T 3L9, Canada, Email: Dominick.Amato@sinaihealthsystem.ca

Received: 25 April, 2019; Accepted: 03 May, 2019; Published: 06 May, 2019

Keywords: Gaucher disease; Bone; Miglustat; Enzyme replacement therapy; Substrate reduction therapy 
Amato D (2019) Improvements in Bone Pain and Patient Quality of Life in Adults with Gaucher Disease During Substrate Reduction Therapy: A Case Series

es to become measurable [7-10]. SRTs act by reducing the amount of substrate, thereby allowing the patient's reduced exogenous enzyme level to process the reduced substrate.

Objective changes in bone abnormalities in GD1 are measured by magnetic resonance imaging (MRI), dual X-ray absorptiometry (DXA) measurement of bone mineral density (BMD), and/or bone marrow burden (BMB) score [11-13]. Subjective improvements in bone pain may or may not occur after starting treatment with either ERT or SRT. In particular, the effects of miglustat on bone in GD1 patients have been reported based on an analysis of data from three multinational open-label clinical trials [14], long-term follow up studies [15-17], and anecdotal reports [18, 19]. Reductions in bone pain and increases in BMD have been observed between 6 months and 2 years after starting miglustat treatment [14]. More recently, long-term follow-up data collected over 12 years in a Spanish GD1 cohort showed that the majority of patients with available bone data (74.4\%) reported an overall reduction in chronic bone pain [15]. Significant bilateral increases in BMD were also seen.

Here we describe three GD1 patients in whom bone manifestations responded remarkably to treatment with the SRT, miglustat. In two of the patients the responses occurred after failure to respond to relatively high doses of ERT. In the third, needle phobia led to miglustat being used as the initial treatment. Psychosocial history was normal in all three patients.

\section{Case Presentation}

\section{Patient 1}

A female patient born in 1979 was diagnosed with type 1 GD at 4 years of age, having presented with marked hepatosplenomegaly. She underwent splenectomy aged 7 years because of worsening organomegaly and thrombocytopenia. Genetic testing at that time established a GD1 genotype of N370S/L444P. Both parents were heterozygous for these mutations; ancestry is Anglo-Saxon. At age 9 years the patient began having bone crises, mainly localized to the hips, and developed avascular necrosis (AVN) in both hips. In 1994, aged 14 years, she was commenced on intravenous alglucerase $30 \mathrm{U} / \mathrm{kg}$ once every 2 weeks. Shortly thereafter she was switched to the same dose of the recombinant product, imiglucerase, after it became available. Physical examination showed the splenectomy scar but was otherwise normal.

After two attacks of cholecystitis, the patient underwent an uneventful laparoscopic cholecystectomy in 2001. Although she did not have any further acute bone crises, she experienced chronic hip pain that showed minimal response to nonsteroidal anti-inflammatory drugs (NSAIDs) or continued ERT. The dose of imiglucerase was there- fore increased gradually to the maximum level of $63 \mathrm{U} / \mathrm{kg}$ every 2 weeks, but still no effect on her bone symptoms was seen. Due to ongoing and progressive hip pain, total hip arthroplasty was performed in 2004 on the left side and later in 2006 on the right side. Between 2009 and 2013, the patient remained on imiglucerase at $63 \mathrm{U} / \mathrm{kg}$ but despite this high-dose therapy she began to have increasing pains in the right knee, right shoulder, left ankle, lower back, and femora. In early 2013 she developed a bone crisis in the right knee. She had been employed as a personal support worker but had to leave that post because of bone pains and fatigue.

In May 2013 the patient discontinued imiglucerase and started on miglustat therapy, with gradual increases in dose from $100 \mathrm{mg}$ q.d. to $100 \mathrm{mg}$ t.i.d. over a period of several weeks and counselling with regard to a low-carbohydrate diet. Over the next year she showed marked symptomatic improvement. While her bone pains have not disappeared, they decreased in terms of both frequency and severity. Pain assessments on a visual analogue scale (VAS) [20,21] indicate substantial improvement (Table 1). She has now returned to work and reports that her quality of life has improved greatly. Her self-ratings of bodily pain, emotional well-being and physical function, assessed with the use of the Short-Form 36 (SF-36) questionnaire [22], which is filled in by each patient at each clinic visit, indicate notable improvements (Table 1).

Left femur and lumbar spine dual X-Ray absorptiometry (DXA), bone mineral density (BMD) measurements and skeletal MRI assessments showed little change during miglustat therapy (Table 2). While not assessed prior to treatment, her BMB score, measured according to the method of Maas et al [23], was 10, indicating moderate to high bone marrow involvement, at last follow up.

\section{Patient 2}

A 59-year-old female patient was diagnosed at age 17 years after presenting with splenomegaly and thrombocytopenia. Genetic testing revealed a genotype of N370S/H162P. Ancestry is a mixture of Scottish, German, French-Canadian, and native Canadian. One sister has mild Gaucher disease and has not required treatment to date. Two brothers have children with Smith-Lemli-Opitz syndrome (7-dehydrocholesterol reductase deficiency). The patient was lost to follow-up for several years, but re-presented aged 28 years with easy bruising, left hip pain, mild splenomegaly and a platelet count of 68 $\mathrm{x} 10^{9} / 1$. She was subsequently lost to follow up again but returned in 2002 aged 44 years with constant left hip and lower back pain. She also complained of easy bruising and marked fatigue. Her platelets had dropped to $28 \times 10^{9} / \mathrm{l}$, and her plasma chitotriosidase (ChT, a marker of disease activity in GD) was $3,840 \mathrm{nmol} / \mathrm{h} / \mathrm{mL}$ (normal range $4-120 \mathrm{nmol} / \mathrm{h} / \mathrm{mL}$ ). Physical examination was normal, with 
Amato D (2019) Improvements in Bone Pain and Patient Quality of Life in Adults with Gaucher Disease During Substrate Reduction Therapy: A Case Series

no palpable organomegaly.

The patient started on biweekly infusions of imiglucerase at 40U/ $\mathrm{kg}$ in 2002. Although her platelet count rose into the normal range, the hip and back pains continued to worsen. She underwent total left hip arthroplasty due to AVN in 2005, after which there was initial improvement. However, she began having pains in other bony areas that were treated with low-dose hydromorphone.

In 2007, the patient enrolled in clinical trial OGT-918-011, a longterm study with miglustat $100 \mathrm{mg}$ t.i.d. After 3 months her bone pains were reduced, but she developed tremors. A reduction of miglustat dosage led to a reduction in tremors, but simultaneously her bone pain recurred. She was therefore removed from the trial and imiglucerase treatment was re-instituted at a dose of $40 \mathrm{U} / \mathrm{kg}$ every 2 weeks. In 2009 she suffered a bone crisis, and in 2010 received four monthly doses of pamidronate, but with no effect. Due mainly to the global shortage of imiglucerase she then switched to velaglucerase at $60 \mathrm{U} /$ $\mathrm{kg}$, gradually increasing to $70 \mathrm{U} / \mathrm{kg}$ every 2 weeks. Again, this ERT had little effect on her chronic bone pains or use of narcotic analgesics.

In 2014, miglustat 100 q.d. was added to the biweekly infusions of velaglucerase, with the miglustat dose gradually increased to $100 \mathrm{mg}$ t.i.d. The doses of both medications were titrated to give maximal benefit with minimal adverse effects [19]. Over the following 2 years the dosage of velaglucerase was gradually decreased until eventually velaglucerase was discontinued at the end of 2016. Miglustat doses were decreased to $100 \mathrm{mg}$ b.i.d. but have recently been increased back up to $100 \mathrm{mg}$ t.i.d. Her current medications include miglustat t.i.d., weekly alendronate, daily vitamin $\mathrm{D}$, and small amounts of hydromorphone.

The patient's quality of life is currently much improved compared with the period between 2002 and 2013, during which her treatment centred mostly on ERT. Subjective self-assessments indicated that her perceived pain was approximately halved during miglustat treatment (Table 1). She also reported modest improvements in bodily pain, emotional well-being and physical function. Objective bone assessments have not yet indicated any notable degree of change, with $\mathrm{BMB}$ score remaining stable and moderate, BMD appearing normal and no change in left femur infarcts (Table 2).

\section{Patient 3}

A 48-year-old male patient born in 1969 was diagnosed with GD at the age of 11 years after presenting with marked splenomegaly and subsequently undergoing splenectomy. His GD genotype is R463C/ L105R. His parents were not available for testing, but family history was non-contributory. Ethnicity consists of Romanian and native Canadian. He first presented in our clinic aged 30 years, at which time his peripheral blood counts were normal, his liver edge was $7.5 \mathrm{~cm}$ below the right costal margin (indicating moderate hepatomegaly), the splenectomy scar was present, and his beta-glucosidase level was $1.0 \mathrm{ng} / \mathrm{h} / \mathrm{mg}$ protein (normal level $8-16 \mathrm{ng} / \mathrm{h} / \mathrm{mg}$ protein). Clinical work-up at that time showed a normal complete blood count, elevated plasma ferritin, and Erlenmeyer-flask deformity of the lower femurs.

The patient was lost to follow-up for 5 years but reappeared briefly in 2007/2008 showing no major changes in clinical phenotype. He then did not return until 2012, at which point he was mildly anaemic $(\mathrm{Hb} 129 \mathrm{~g} / \mathrm{L})$ and had a mild polyclonal gammopathy, hepatomegaly (liver volume $3,200 \mathrm{~cm}^{3}$ on abdominal CT scan vs. expected volume of 2,500 $\left.\mathrm{cm}^{3}\right)$, and highly elevated plasma ChT $(10,295 \mathrm{nmol} / \mathrm{h} /$ $\mathrm{mL})$. An unexpected finding was the presence of a $1.9-\mathrm{cm}$ lesion in the right kidney for which he underwent a partial right nephrectomy that confirmed a diagnosis of renal-cell carcinoma. Post-op urological examination has shown no evidence of recurrence or metastasis.

Over the next few years the patient developed type 2 diabetes mellitus for which he was started on metformin. He also developed increasing bone pains in the mid-back, both knees, and toes. Bone MRI showed patchy infiltration of the femora and lumbar spine. He had a BMB score of 5 (indicating mild bone marrow involvement), and DXA scanning showed a DXA-BMD level within the expected range for his age. As the patient has a lifelong extreme aversion to needles, he was started on oral miglustat therapy in late-2017, with doses increased gradually from $100 \mathrm{mg}$ o.d. to $100 \mathrm{mg}$ t.i.d. over a period of 6 weeks. He experienced mild bloating and flatulence during the first month of miglustat therapy, but no gastrointestinal or other adverse effects have been reported since then.

The patient's perceived bone pains have improved greatly, with substantial improvements recorded on the pain VAS and SF-36 bodily pain domain (Table 1).

\begin{tabular}{|l|c|c|c|c|c|c|}
\hline \multirow{2}{*}{ Parameter } & \multicolumn{2}{|c|}{ Patient 1 } & \multicolumn{2}{c|}{ Patient 2 } & \multicolumn{2}{c|}{ Patient 3 } \\
\cline { 2 - 7 } & $\begin{array}{c}\text { Pre-treat- } \\
\text { ment }\end{array}$ & $\begin{array}{c}\text { Post treat- } \\
\text { ment (1 yr) }\end{array}$ & $\begin{array}{c}\text { Pre-treat- } \\
\text { ment }\end{array}$ & $\begin{array}{c}\text { Post treat- } \\
\text { ment (2 yr) }\end{array}$ & $\begin{array}{c}\text { Pre-treat- } \\
\text { ment }\end{array}$ & $\begin{array}{c}\text { Post treatment (10 } \\
\text { mo.) }\end{array}$ \\
\hline VAS $^{*}$ & 8.0 & 2.0 & 6.7 & 3.7 & 7.0 & 0 \\
\hline Bodily pain $^{\dagger}$ & 34.1 & 38.9 & 54.2 & 65.7 & 28.3 & 63.7 \\
\hline Role emotional $^{\dagger}$ & 60.6 & 74.1 & 44.4 & 55.6 & 54.6 & 62.0 \\
\hline Physical functioning $^{\dagger}$ & 32.3 & 45.0 & 54.6 & 62.0 & 57.9 & 60.0 \\
\hline
\end{tabular}


Amato D (2019) Improvements in Bone Pain and Patient Quality of Life in Adults with Gaucher Disease During Substrate Reduction Therapy: A Case Series

*Visual analogue scale, where lower scores indicate improvement; $\uparrow$ derived from SF-36 assessments filled in by each patient at each clinic visit (increased scores represent improvement).

Table 1: Changes in subjective pain and quality-of-life patient parameters in three patients before and after treatment with miglustat

He is in frequent e-mail contact, reporting that he is mostly pain free, which he describes as a "a joy", as reflected by improvements in SF-36 emotional well-being and physical scores. No changes in objective bone parameters have been recorded (Table 2).

\begin{tabular}{|c|c|c|c|c|c|c|}
\hline \multirow[t]{2}{*}{ Parameter } & \multicolumn{2}{|c|}{ Patient 1} & \multicolumn{2}{|c|}{ Patient 2} & \multicolumn{2}{|c|}{ Patient 3} \\
\hline & Pre-treatment & $\begin{array}{c}\text { Post treatment } \\
\text { (1 yr) }\end{array}$ & Pre-treatment & $\begin{array}{l}\text { Post treat- } \\
\text { ment ( } 2 \text { yr) }\end{array}$ & Pre-treatment & $\begin{array}{c}\text { Post treatment (10 } \\
\text { mo.) }\end{array}$ \\
\hline MRI & $\begin{array}{l}\text { EF, MI, right } \\
\text { distal femur } \\
\text { infarct }\end{array}$ & $\begin{array}{l}\text { More prom- } \\
\text { inent MI, } \\
\text { otherwise } \\
\text { unchanged }\end{array}$ & $\begin{array}{c}\text { Multiple } \\
\text { LF infarcts }\end{array}$ & No change & EF, MI & No change \\
\hline BMB score & ND & 10 & 5 & 6 & 5 & 5 \\
\hline BMD (DXA) & $\begin{array}{l}\text { LS -1.1; } \\
\text { LF -2.2 }\end{array}$ & $\begin{array}{l}\text { LS -1.3; } \\
\text { LF -2.3 }\end{array}$ & Normal & Normal & Normal & Pending \\
\hline
\end{tabular}

Table 2: Changes in objective bone parameters in three patients before and after treatment with miglustat

\section{$\underline{\text { Discussion }}$}

In this case series, notable and rapid improvements in subjective bone outcomes (mainly bone pain) were observed following transition from long-term ERT to miglustat monotherapy in patients 1 and 2, and after de novo initiation of miglustat treatment in patient 3. Patients 1 and 2 had not previously shown any change in bone status during 14-19 years of previous ERT, which is consistent with previous data showing relatively little effect of long-term ERT on bone status in GD $[1,9,24]$. Only minimal effects on objective bone parameters (DXA-BMD, BMB and MRI findings) were observed during the observation period. Importantly, all patients reported substantial effects on key quality of life aspects (emotional wellbeing and physical function). These findings should be considered alongside the prevailing focus on patient-centric outcomes data, which are important at the clinical practice level as well as in clinical trials, particularly in rare diseases such as GD1 where study patient numbers tend to be low $[25]$.

The reductions in bone pain reported here are in line with previous data from miglustat clinical studies. In a long-term follow-up study of 63 Spanish miglustat-treated GD1 patients, the majority (74\%) of those with available bone data reported an overall reduction in chronic bone pain [15]. Based on a pooled analysis of data from three multinational, open-label clinical trials assessing the efficacy of miglustat, Pastores, et al. [14] reported that over $80 \%$ of patients had no bone pain over 2 years of miglustat monotherapy, regardless of previous ERT or spleen status. Further, among treatment-naïve patients, reports of bone pain decreased from $46 \%$ to $14 \%$, and among those previously on ERT this proportion decreased from $76 \%$ to $19 \%$ [14]. Notable reductions (from 12\% to 3\%) in requirements for analgesic medication due to GD1-related bone pain have also been reported in ERT-naïve patients after initiation of miglustat [26]. This seems to reflect improvements observed in patients 2 and 3 in the current case series. It is also noteworthy that in patient 2 , bone pains were reduced after just 3 months of initial miglustat therapy and stopping treatment for a period of time led to a return of her pain.

Changes in objective bone parameters in our patients were generally small. Little change was observed in either DXA-BMD or BMB during 1-4 years of miglustat therapy, although no baseline (pre-miglustat) $\mathrm{BMB}$ score was available in patient 3 , who showed the greatest degree of burden. In contrast, early and sustained increases in both lumbar spine and femoral neck BMD during miglustat monotherapy have previously been reported, with significant increases from baseline evident at 6,12 and 24 months [14]. In the Spanish study of bone changes during miglustat therapy, statistically significant improvements in both BMB and BMD were observed during up to 12 years of treatment [15]. The reasons for the relative lack of change in $\mathrm{BMB}$ or BMD in patients 1 (treated for approximately 5 years) and 2 (treated for 2 years on miglustat monotherapy) in the current case series are not clear. However, patient 3 has only received miglustat for approximately 1 year to date so could not really be expected to show notable changes.

It has been suggested that miglustat may exert greater effects on bone 
Amato D (2019) Improvements in Bone Pain and Patient Quality of Life in Adults with Gaucher Disease During Substrate Reduction Therapy: A Case Series

in GD compared with ERT due to its physicochemical properties, which enable a wide distribution throughout body tissues, including bone $[14,27]$. Glycosphingolipids are involved in osteoclast differentiation, and miglustat, an inhibitor of glucosylceramide synthase, may have a direct effect on osteoclastogenesis within bone marrow $[14,28,29]$. Monocyte- and T cell-mediated inflammatory pathways may also be involved in osteoclast modulation in GD, and effects of GD therapy on this aspect have been proposed [30]. Overall though, the exact mechanism of action of miglustat on bone remodelling and its downstream effect on perceived bone pain require further study. Further, it remains to be determined whether effects on bone parameters might indicate a form of class effect for glucosylceramide synthase inhibitors (including miglustat and eliglustat), as positive effects have also been reported in long-term clinical trials with eliglustat [31].

The two most common adverse effects of miglustat are gastrointestinal (GI) disturbances and tremor. The gradual introduction of the medication with slow increases up to the recommended dose over a period of several weeks, combined with a low-carbohydrate diet, has in our Clinic minimized the GI effects. Although some of our patients have experience tremor on miglustat, this was minimal in patient 1 and absent in patients 2 and 3 .

\section{Conclusions}

In conclusion, consistent with previous published data, miglustat improved subjective bone outcomes and QoL in our three patients who had very different medical and treatment histories. It is important to note that remarkable improvements were seen in two patients despite a previous lack of response of bone-related outcomes to long-term ERT given at fairly high doses. In addition, patient-reported outcomes (Table 1) will likely play a greater role in clinical publications in the future. Relatively little or no effects on objective bone parameters (e.g., $\mathrm{BMB}$ and $\mathrm{BMD}$ ) were observed during miglustat therapy but, considering previous published data, the possibility of greater changes with longer-term miglustat treatment cannot be ruled out.

\section{$\underline{\text { Abbreviations }}$}

BMB: Bone marrow burden; BMD: Bone mineral density; EF: Erlenmeyer flask deformity; ERT: Enzyme replacement therapy; GD1: Gaucher disease type 1; MI: Marrow infarction; SRT: Substrate reduction therapy, VAS: Visual analogue scale

\section{$\underline{\text { Declarations }}$}

\section{Ethics approval and consent to participate}

Not applicable.

\section{Consent for publication}

Written informed consent was obtained from the patients for publication of this case series and any accompanying images. A copy of the written consent is available for review by the Editor-in-Chief of this journal.

\section{Availability of data and materials}

Not applicable.

\section{Competing Interests}

DA has received research and operating grants, honoraria for speaking, and travel funds from Actelion Pharmaceuticals, Genzyme Corporation, Protalix Biopharmaceuticals, and Shire Human Genetic Therapies.

\section{Funding}

No funding was received for the conduct of this case series.

\section{$\underline{\text { Author contributions }}$}

As corresponding author, DA coordinated the specialist clinical care of the patient, wrote the first draft of the manuscript, was involved at all writing stages of the manuscript content, and approved the final draft of the manuscript for submission. MAP coordinated patient care, contributed to the writing, and approved the final draft.

\section{Acknowledgements}

Matthew Reilly PhD from InTouch Medical Ltd provided medical writing support in the preparation of this case report, paid for by Actelion Pharmaceuticals Ltd.

\section{References}

1. Mankin HJ, Trahan CA, Barnett NA, Laughead J, Bove CM, et al. (2006) A questionnaire study for 128 patients with Gaucher disease. Clin Genet 69: 209-217.

2. Wenstrup RJ, Roca-Espiau M, Weinreb NJ, Bembi B (2002) Skeletal aspects of Gaucher disease: a review. Br J Radiol 75: A212. [Crossref]

3. Mikosch P, Hughes D (2010) An overview on bone manifestations in Gaucher disease. Wien Med Wochenschr 160: 609-624. [Crossref]

4. Cox TM (2010) Recommendations for treating patients with Gaucher disease with emerging enzyme products. Blood Cells Mol Dis 44: 84-85. [Crossref] 
Amato D (2019) Improvements in Bone Pain and Patient Quality of Life in Adults with Gaucher Disease During Substrate Reduction Therapy: A Case Series

5. Pastores GM, Giraldo P, Cherin P, Mehta A (2009) Goal-oriented therapy with miglustat in Gaucher disease. Curr Med Res Opin 25: 23-37. [Crossref]

6. Belmatoug N, Di Rocco M, Fraga C, Giraldo P, Hughes D, et al. (2017) Management and monitoring recommendations for the use of eliglustat in adults with type 1 Gaucher disease in Europe. Eur J Intern Med 37: 25-32. [Crossref]

7. Weinreb NJ, Goldblatt J, Villalobos J, Charrow J, Cole JA, et al. (2013) Long-term clinical outcomes in type 1 Gaucher disease following 10 years of imiglucerase treatment. J Inherit Metab Dis 36: 543-553. [Crossref]

8. Wenstrup RJ, Kacena KA, Kaplan P, Pastores GM, PrakashCheng A, et al. (2007) Effect of enzyme replacement therapy with imiglucerase on BMD in type 1 Gaucher disease. J Bone Miner Res 22: 119-126. [Crossref]

9. de Fost M, van Noesel CJ, Aerts JM, Maas M, Poll RG, et al. (2008) Persistent bone disease in adult type 1 Gaucher disease despite increasing doses of enzyme replacement therapy. Haematologica 93: 1119-1120. [Crossref]

10. de Fost M, Hollak CE, Groener JE, Aerts JM, Maas M, et al. (2006) Superior effects of high-dose enzyme replacement therapy in type 1 Gaucher disease on bone marrow involvement and chitotriosidase levels: a 2-center retrospective analysis. Blood 108: 830-835. [Crossref]

11. Vom Dahl S, Poll L, Di Rocco M, Ciana G, Denes C, et al. (2006) Evidence-based recommendations for monitoring bone disease and the response to enzyme replacement therapy in Gaucher patients. Curr Med Res Opin 22: 1045-1064. [Crossref]

12. Maas M, van Kuijk C, Stoker J, Hollak CEM, Akkerman EM, et al. (2003) Quantification of bone involvement in Gaucher disease: MR imaging bone marrow burden score as an alternative to Dixon quantitative chemical shift MR imaging-initial experience. Radiol 229: 554-561. [Crossref]

13. Komninaka V, Kolomodi D, Christoulas D, Marinakis T, Papatheodorou A, et al. (2015) Evaluation of bone involvement in patients with Gaucher disease: a semi-quantitative magnetic resonance imaging method (using ROI estimation of bone lesion) as an alternative method to semi-quantitative methods used so far. Eur J Haematol 95: 342-351. [Crossref]

14. Pastores GM, Elstein D, Hrebicek M, Zimran A (2007) Effect of miglustat on bone disease in adults with type 1 Gaucher disease: a pooled analysis of three multinational, open-label studies. Clin
Ther 29: 1645-1654. [Crossref]

15. Giraldo P, Andrade-Campos M, Alfonso P, Irun P, Atutxa K, et al. (2016) Twelve years of experience with miglustat in the treatment of type 1 Gaucher disease: The Spanish ZAGAL project. Blood Cells Mol Dis 107: 30133-30134. [Crossref]

16. Giraldo P, Alfonso P, Atutxa K, Fernandez-Galan MA, Barez A, et al. (2009) Real-world clinical experience with long-term miglustat maintenance therapy in type 1 Gaucher disease: the ZAGAL project. Haematologica 94: 1771-1775. [Crossref]

17. Giraldo P, Latre P, Alfonso P, Acedo A, Alonso D, et al. (2006) Short-term effect of miglustat in every day clinical use in treatment-naive or previously treated patients with type 1 Gaucher's disease. Haematologica 91: 703-706.

18. Mikosch P, Reed M, Baker R, Holloway B, Berger L, et al. (2008) Changes of bone metabolism in seven patients with Gaucher disease treated consecutively with imiglucerase and miglustat. Calcif Tissue Int 83: 43-54. [Crossref]

19. Amato D, Patterson MA (2018) Combined miglustat and enzyme replacement therapy in two patients with type 1 Gaucher disease: two case reports. J Med Case Rep 12: 19. [Crossref]

20. Reips UD, Funke F (2008) Interval-level measurement with visual analogue scales in Internet-based research: VAS Generator. Behav Res Methods 40: 699-704.

21. Grant S, Aitchison T, Henderson E, Christie J, Zare S, et al. (1999) A comparison of the reproducibility and the sensitivity to change of visual analogue scales, Borg scales, and Likert scales in normal subjects during submaximal exercise. Chest 116: 12081217.

22. Ware JE, Sherbourne CD (1992) The MOS 36-item short-form health survey (SF-36). I. Conceptual framework and item selection. Med Care 30: 473-483.

23. Maas M, van Kuijk C, Stoker J, Hollak CE, Akkerman EM, et al. (2003) Quantification of bone involvement in Gaucher disease: MR imaging bone marrow burden score as an alternative to Dixon quantitative chemical shift MR imaging--initial experience. Radiology 229: 554-561. [Crossref]

24. Weinreb NJ, Charrow J, Andersson HC, Kaplan P, Kolodny EH, et al. (2002) Effectiveness of enzyme replacement therapy in 1028 patients with type 1 Gaucher disease after 2 to 5 years of treatment: a report from the Gaucher Registry. Am J Med 113: 112-119. 
Amato D (2019) Improvements in Bone Pain and Patient Quality of Life in Adults with Gaucher Disease During Substrate Reduction Therapy: A Case Series

25. Weldring T, Smith SM (2013) Patient-Reported Outcomes (PROs) and Patient-Reported Outcome Measures (PROMs). Health Serv Insights 6: 61-68. [Crossref]

26. Kuter DJ, Mehta A, Hollak CE, Giraldo P, Hughes D, et al. (2013) Miglustat therapy in type 1 Gaucher disease: clinical and safety outcomes in a multicenter retrospective cohort study. Blood Cells Mol Dis 51: 116-124. [Crossref]

27. Treiber A, Morand O, Clozel M (2007) The pharmacokinetics and tissue distribution of the glucosylceramide synthase inhibitor miglustat in the rat. Xenobiotica 37: 298-314. [Crossref]

28. Reed MC, Schiffer C, Heales S, Mehta AB, Hughes DA (2018) Impact of sphingolipids on osteoblast and osteoclast activity in Gaucher disease. Mol Genet Metab 124: 278-286. [Crossref]
29. Ersek A, Xu K, Antonopoulos A, Butters TD, Santo AE, et al. (2015) Glycosphingolipid synthesis inhibition limits osteoclast activation and myeloma bone disease. J Clin Invest 125: 22792292. [Crossref]

30. Mucci JM, Cuello MF, Kisinovsky I, Larroude M, Delpino MV, et al. (2015) Proinflammatory and proosteoclastogenic potential of peripheral blood mononuclear cells from Gaucher patients: Implication for bone pathology. Blood Cells Mol Dis 55: 134-143. [Crossref]

31. Mistry PK, Lukina E, Ben Turkia H, Shankar SP, Baris H, et al. (2017) Outcomes after 18 months of eliglustat therapy in treatment-naive adults with Gaucher disease type 1: The phase $3 \mathrm{EN}$ GAGE trial. Am J Hematol 92: 1170-1176. [Crossref]

Copyright: (C) 2019 Amato D. This is an open-access article distributed under the terms of the Creative Commons Attribution License, which permits unrestricted use, distribution, and reproduction in any medium, provided the original author and source are credited. 